\title{
LINKS NOT CONCORDANT TO BOUNDARY LINKS
}

\author{
CHARLES LIVINGSTON
}

(Communicated by Frederick R. Cohen)

\begin{abstract}
Casson-Gordon invariants are used to prove that certain links in $S^{3}$ are not concordant to boundary links. These examples were first described by Cochran and Orr.
\end{abstract}

Tim Cochran and Kent Orr recently announced the construction of 2 component links in $S^{3}$ with the property that, although all the Milnor $\bar{\mu}$-invariants vanish, the links are not concordant to boundary links [CO]. They also provide examples of higher dimensional links of two components which are not concordant to boundary links. Their 3-dimensional examples include the links $L_{m}(m>0)$ illustrated in Figure 1 (see p. 1131). Both bands on the Seifert surface for $K_{1}$ are untwisted; that is, the Seifert form vanishes on the homology classes represented by $x$ and $y$. They let $K$ be the trefoil. The knot $K_{1}$ (with $m=1$ ) was used in my paper with Gilmer [GL1] as an example of a slice knot that is algebraically doubly null concordant, but not doubly null concordant. The results of $[\mathrm{CO}]$ and $[\mathrm{GL} 1]$ are related. We will show here that the result that $L_{m}$ is not concordant to a boundary link follows readily from the results of Gilmer [G] on which the work in [GL1] was based. Gilmer's work was based on earlier work on knot concordance by Levine [L] and Casson and Gordon [CG1, CG2].

Danny Ruberman [R1] generalized the work of [GL1] to higher dimensions. (See also [R2] and [Sm] for corrections to [R1].) His observation that the Casson-Gordon invariants apply to higher dimensional slice problems is relevant here. However, a more careful analysis of the Casson-Gordon invariants is needed to prove that the high dimensional examples of [CO] are not concordant to boundary links. That analysis will be presented in a separate paper being written with Pat Gilmer [GL2].

Tim Cochran has informed me that he is also investigating the relationship between the work announced in [CO] and the Casson-Gordon invariant.

The rest of this note is devoted to the proof that $L_{m}$ is not concordant to a boundary link.

Received by the editors August 30, 1989.

1980 Mathematics Subject Classification (1985 Revision). Primary 57M25.

Key words and phrases. Link concordance, boundary links, Casson-Gordon invariant. 
Pat Gilmer proved that if a slice knot $K$ in $S^{3}$ bounds a genus 1 Seifert surface $F$, then there is a simple closed curve $J$ on $F$ representing a primitive element in $H_{1}(F)$ and for which Tristram $p$-signatures, $\sigma_{(s / p)}(J)$, vanish for all $0<s<p$, where $p$ is any prime dividing the order of the first homology of the 2-fold cyclic cover of $S^{3}$ branched over $K$. Gilmer produced $J$ as follows. Suppose that $K$ bounds a disk $D$ in $B^{4}$. The surface $F \cup D$ bounds a 3-manifold $R$ in $B^{4}$. Let $i$ denote the inclusion of $F$ into $R$, and let $H$ denote $i_{*}^{-1}\left(\operatorname{Tor}\left(H_{1}(R)\right)\right.$. Gilmer showed that $H$ is a 1-dimensional summand of $H_{1}(F)$ and that if $J$ represents a generator of $H$ then it has the desired signature properties.

To proceed, consider the link $L=K_{1} \cup K_{2} . K_{1}$ is clearly slice. (Perform ambient surgery on the unknotted curve $y$ on the evident Seifert surface $F_{1}$.) We will show that if $L$ is concordant to a boundary link then $K_{1}$ bounds a disk $D$ in $B^{4}$ with the property that $F_{1} \cup D$ bounds a 3-manifold $R$ for which the generator of the summand $H$ described above is represented by $x$.

The construction of $D$ and $R$ is done in two steps. First, suppose that $L$ is concordant in $S^{3} \times[0,1]$ to the boundary link $L^{\prime}=K_{1}^{\prime} \cup K_{2}^{\prime}$ bounding disjoint surfaces $F_{1}^{\prime}$ and $F_{2}^{\prime}$. Denote the two components of the concordance $C_{1}$ and $C_{2}$. The following transversality argument shows that the closed surface $F_{1} \cup F_{1}^{\prime} \cup C_{1}$ bounds a 3-manifold $R_{1}$ in $S^{3} \times[0,1]$ with $R_{1}$ disjoint from $C_{2}$. (This was stated without proof in $[\mathrm{S}]$.)

Let $\nu$ denote tubular neighborhood. There is a map $p$ of $\left(S^{3} \times\{0\}-\right.$ $\left.\nu\left(K_{1} \cup K_{2}\right)\right) \cup\left(S^{3} \times\{1\}-\nu\left(K_{1}^{\prime} \cup K_{2}^{\prime}\right)\right) \cup \partial\left(\nu\left(C_{2}\right)\right)$ to $S^{1}$ such that $p$ is transverse to $1, p^{-1}(1)=F_{1} \cup F_{1}^{\prime}$ and $p^{-1}(-1)$ contains $\partial\left(\nu\left(C_{2}\right)\right)$. Constructing $R_{1}$ via transversality depends on extending $p$ to $S^{3} \times[0,1]-\nu\left(C_{1} \cup C_{2}\right)$.

Homotopy classes of maps of a space $X$ to $S^{1}$ correspond to elements of $H^{1}(X)$. The restriction map of

$$
H^{1}\left(S^{3} \times[0,1]-\nu\left(C_{1} \cup C_{2}\right)\right)
$$

to

$$
H^{1}\left(\left(S^{3} \times\{0\}-\nu\left(K_{1} \cup K_{2}\right)\right) \cup\left(S^{3} \times\{1\}-\nu\left(K_{1}^{\prime} \cup K_{2}^{\prime}\right)\right) \cup \partial\left(\nu\left(C_{2}\right)\right)\right)
$$

is a map of $\mathbf{Z}^{2}$ to $\mathbf{Z}^{3}$ with image consisting of those elements that agree on the meridians of $K_{1}$ and $K_{1}^{\prime}$. Our map $p$ represents a class in the image, and hence $p$ is the restriction of a map as desired.

Let $D_{1}$ be a slice disk for $K_{1}^{\prime}$ in $B^{4}$, and let $R_{1}^{\prime}$ be a 3-manifold bounded by $F_{1}^{\prime} \cup D_{1}$ in $B^{4}$. Form the union of $S^{3} \times[0,1]$ with $B^{4}$ identifying $S^{3} \times\{1\}$ with $\partial B^{4}$. The union of $C_{1}$ with $D_{1}$ forms a slice disk $D$ for $K_{1}$. The union of $R_{1}$ and $R_{1}^{\prime}$ forms a 3-manifold $R$ bounded by $F_{1} \cup D$. Note that $R$ is in the complement of the connected surface $E=C_{2} \cup F_{2}^{\prime}$ bounded by $K_{2}$.

If an element $z$ in $H_{1}\left(F_{1}\right)$ represents torsion in $H_{1}(R)$, it is also torsion in $H_{1}\left(B^{4}-E\right)$. The element $z$ can be written as $z=a x+b y$. In $H_{1}\left(B^{4}-E\right)$, 


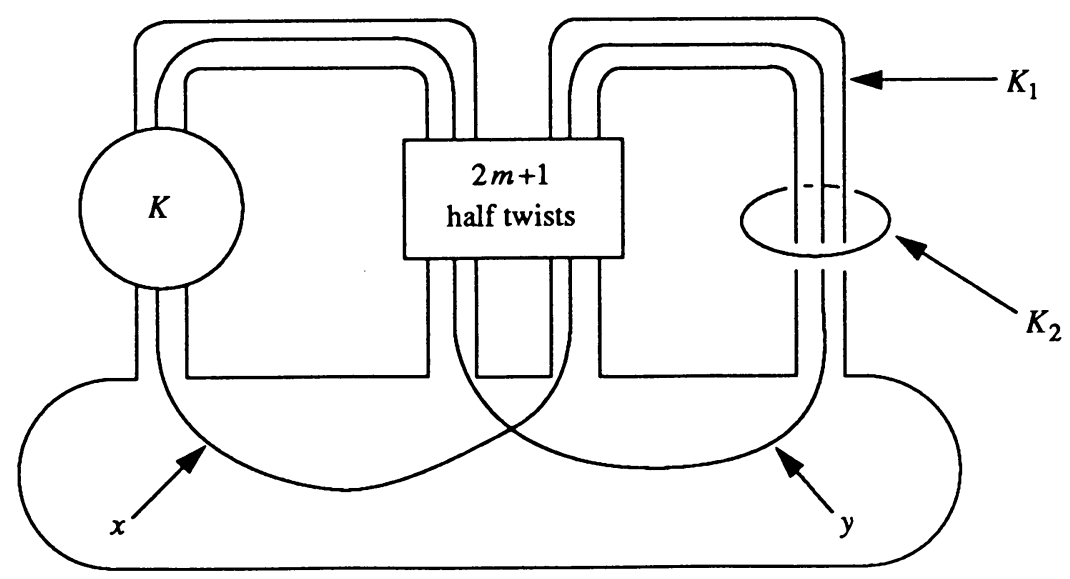

FIGURE 1

$x$ represents zero and $y$ an element of infinite order, in fact the generator. (Linking numbers of $x$ and $y$ with $E$ in $B^{4}$ equal their linking numbers with $K_{2}$ in $S^{3}$.) It follows that $b$ is 0 , and hence that $H$ is generated by the class represented by $x$.

The curve $x$ is of the same knot type as $K$. The order of the homology of the 2-fold cyclic branched cover of $S^{3}$ branched over $K_{1}$ is $(2 m+1)^{2}$. Hence, as long as $\sigma_{(s / p)}(K) \neq 0$ for some prime divisor $p$ of $(2 m+1)$ and $0<s<p$, $L$ will not be concordant to a boundary link. The trefoil works for all $m>0$.

\section{REFERENCES}

[CO] T. Cochran and K. Orr, Not all links are concordant to boundary links, preprint.

[CG1] A. Casson and C. McA. Gordon, Cobordism of classical knots, preprint, Orsay; reprinted in $A$ la recherche de la topologie Perdue (A. Marin and L. Guillou, eds.), Progress in Math. vol. 62, Birkhauser, 1986.

[CG2] _- On slice knots in dimension three, Proc. Sympos. Pure Math. XXX (1978), Part two, 39-53.

[G] P. Gilmer, Slice knots in $S^{3}$, Quart. J. Math. Oxford 39 (1983), 305-322.

[GL1] P. Gilmer and C. Livingston, On embedding 3-manifolds in 4-space, Topology 22 (1983), 241-252.

[GL2] _ The Casson-Gordon invariant and link concordance, preprint.

[L] J. Levine, Knot cobordism groups in codimension two, Comment. Math. Helv. 44 (1969), 229-244.

[R1] D. Ruberman, Doubly slice knots and the Casson-Gordon invariants, Trans. Amer. Math. Soc. 279 (1983), 569-588.

[R2] Casson-Gordon invariants and high-dimensional knot theory, Trans. Amer. Math. Soc. 306 (1988), 579-596.

[S] N. Sato, Cobordisms of semi-boundary links, Topology Appl. 18 (1984), 225-234.

[Sm] L. Smolinsky, Casson-Gordon invariants of some 3-fold branched covers of knots, Topology Appl. 31 (1989), 243-252.

Department of Mathematics, Indiana University, Bloomington, Indiana 47405 